\begin{tabular}{|c|c|}
\hline Title & $\begin{array}{l}\text { Risk factors for residual mitral regurgitation after aortic valve replacement in patients with severe aortic valve stenosis } \\
\text { and moderate mitral regurgitation }\end{array}$ \\
\hline Author(s) & $\begin{array}{l}\text { Shingu, Y asushige; Iwano, Hiroyuki; Murakami, Tatsuya; Katoh, Nobuy asu; Ooka, Tomonori; Katoh, Hiroki; Kubota, } \\
\text { Suguru; Matsui, Y oshiro }\end{array}$ \\
\hline Citation & $\begin{array}{l}\text { General thoracic and cardiovascular surgery, } 67,849-854 \\
\text { https://doi.org/10.1007/s11748-019-01110-w }\end{array}$ \\
\hline Issue Date & 2019-10 \\
\hline Doc URL & http:/hdl.handle.net/2115/79360 \\
\hline Rights & $\begin{array}{l}\text { This is a post-peer-review, pre copyedit version of an article published in General thoracic and cardiovascular surgery. } \\
\text { The final authenticated version is avail able online at: https://doi.org } / 10.1007 / \delta 11748-019-01110-\mathrm{w}\end{array}$ \\
\hline Type & article (author version) \\
\hline File Information & Gen Thorac Cardiovasc Surg_67(10)_849.pdf \\
\hline
\end{tabular}

Instructions for use 
Original Cardiovascular

\section{Risk factors for residual mitral regurgitation after aortic valve replacement in patients with severe aortic valve stenosis and moderate mitral regurgitation}

Yasushige Shingu, $\mathrm{MD}, \mathrm{PhD}^{1}$, Hiroyuki Iwano, $\mathrm{MD}, \mathrm{PhD}^{2}$, Tatsuya Murakami, $\mathrm{MD}, \mathrm{PhD}^{3}$, Nobuyasu Katoh, MD, $\mathrm{PhD}^{1}$, Tomonori Ooka, $\mathrm{MD}, \mathrm{PhD}^{1}$, Hiroki Katoh, $\mathrm{MD}, \mathrm{PhD}^{4}$, Suguru Kubota, $\mathrm{MD}, \mathrm{PhD}^{1}$, and Yoshiro Matsui, $\mathrm{MD}, \mathrm{PhD}^{1}$

${ }^{1}$ Department of Cardiovascular and Thoracic Surgery, Faculty of Medicine and Graduate School of Medicine, Hokkaido University, Sapporo, Japan

${ }^{2}$ Department of Cardiovascular Medicine, Faculty of Medicine and Graduate School of Medicine, Hokkaido University, Sapporo, Japan

${ }^{3}$ Department of Thoracic Surgery, Asahikawa City Hospital, Asahikawa, Japan

${ }^{4}$ Emergency and Critical Care Center, Hokkaido University Hospital, Sapporo, Japan

*Corresponding author: Dr. Yasushige Shingu

Department of Cardiovascular and Thoracic Surgery, Faculty of Medicine and Graduate School of Medicine, Hokkaido University

Kita-15, Nishi-7, Kita-Ku, Sapporo 060-8638, Japan

Tel: +81-11-706-6042; Fax: +81-11-706-7612

Email: shingu@huhp.hokudai.ac.jp

Running title: Risk factors for residual MR after SAVR in AS

Key words: aortic valve stenosis, mitral regurgitation

Total word count: 3125 


\begin{abstract}
Objectives: While it was reported that patients with residual moderate mitral regurgitation (MR) after surgical aortic valve replacement (SAVR) had a poorer prognosis than those without it, the risk factors for residual MR have not been fully elucidated. The aim of the study was to evaluate risk factors for residual MR after SAVR.
\end{abstract}

Methods: Of the 222 patients who underwent isolated SAVR from 2001 to 2018, 33 (11 men; age: $74 \pm 7$ years) had functional moderate MR before surgery. The risk factors for residual MR were evaluated by comparing patients with residual moderate MR ( $\mathrm{n}=11,33 \%)$ with those who exhibited improved post-surgery MR ( $\mathrm{n}=22,67 \%)$.

Results: The left atrial diameter was significantly larger in the residual MR group (51 $\pm 7 \mathrm{~mm})$ than in the improved MR group (46 $\pm 5 \mathrm{~mm} ; \mathrm{P}=0.049$ ). The mean pressure gradient at the aortic valve was significantly smaller in the residual MR group (52 $\pm 18 \mathrm{mmHg})$ than in the improved MR group (69 $\pm 22 \mathrm{mmHg} ; \mathrm{P}=0.043)$. A ratio of left atrial diameter $(\mathrm{mm})$ and mean aortic valve pressure gradient (mmHg) greater than 0.9 predicted residual MR with a sensitivity of $70 \%$ and a specificity of 74\% (area under the ROC curve: 0.779; $\mathrm{P}=0.015$ ).

Conclusions: In patients with severe aortic valve stenosis and moderate MR, a high ratio of preoperative left atrial diameter and mean aortic valve pressure gradient would be a parameter predicting residual moderate MR post SAVR. 


\section{Introduction}

The prevalence of moderate aortic valve (AV) disease is as high as $9 \%$ in those older than 80 years (1). AV stenosis (AS) is the most common heart valve disease in the elderly. Functional mitral regurgitation (MR) may accompany AS because of a high left ventricle (LV) to left atrial pressure gradient, LV remodelling, and atrial fibrillation (2). Preoperative moderate MR due to various etiologies was reported to independently predict a higher mortality after isolated surgical AV replacement (SAVR) (3). However, the beneficial effects of correcting moderate MR during AV surgery have not yet been demonstrated. In the guidelines of the American Heart Association, mitral valve repair for patients with chronic, moderate secondary MR who undergo other cardiac surgeries, only has a class IIb recommendation (4). The controversy may stem from possible MR improvement observed after SAVR. In 45\%-82\% of patients with severe AS and moderate functional MR, MR was reported to improve after isolated SAVR (2). In that review, the risk factors for residual MR after surgery were high systolic pulmonary artery pressure (>50 mmHg), large left atrial diameter ( $>50 \mathrm{~mm}$ ), and atrial fibrillation. However, the risk factors that predict residual MR are still debated. The aim of this study was to evaluate risk factors for residual MR after SAVR.

\section{Materials and Methods}

\section{Subjects}

This retrospective study was conducted at a single center (Hokkaido University Hospital). Of the 222 patients who underwent isolated SAVR for severe AS from 2001 to 2018, 33 (15\%) patients (11 men; age, $74 \pm 7$ years) with moderate MR before surgery were included in the study. Subjects with severe MR and any mitral prolapse were excluded from the study. The risk factors for postoperative residual MR were evaluated by comparing patients with residual moderate MR with 
those with improved post-surgery MR. The university ethics committee approved the research protocol (No. 018-0330), and informed consent was waived.

\section{Transthoracic echocardiography}

Commercially available ultrasonic systems including Vivid 7 or Vivid E9 (GE Healthcare, Milwaukee, WI, USA), Aplio XG or Aplio Artida (Toshiba Medical Systems, Tochigi, Japan), and Sonos 5500 or iE33 (Philips Ultrasound, Bothell WA, USA) were used for transthoracic echocardiography. Left ventricular (LV) and atrial diameters were measured from the parasternal long axis view. LV ejection fraction was measured using the biplane method of disks. The main endpoint was MR grade, which was defined by blinded examiners using the regurgitation jet areato-left atrial area ratio (mild, $<20 \%$; moderate, 20-40\%; severe, $>40 \%$ ). Tricuspid regurgitation was graded using the regurgitation jet area (mild, $<5 \mathrm{~cm}^{2}$; moderate, $5-10 \mathrm{~cm}^{2}$; severe, $>10 \mathrm{~cm}^{2}$ ). The mitral valve was visually assessed and judged as thickened if it was obviously thick compared to the tricuspid valve. Calcification was defined as a focal high echoic region with acoustic shadow. Trans-AV gradient was estimated from trans-AV flow velocity by using continuous Doppler imaging. AV area was estimated by a continuous formula, severe AS: area $<1.0 \mathrm{~cm}^{2}$. AV regurgitation was determined using a combination of jet width/outflow tract, pressure half-time, and diastolic reverse flow at the abdominal aorta (mild; moderate; severe) (5).

\section{Aortic valve replacement}

AV replacement was performed as an isolated procedure under cardiac arrest for all the patients. Usual extracorporeal circulation was established with cannulation in the ascending aorta and right atrium. Myocardial protection was performed with antegrade and retrograde cardioplegia every 30 minutes. A bioprosthetic valve was selected in most cases (30/33, 91\%). The valve size was $17 \mathrm{~mm}$ in two, $19 \mathrm{~mm}$ in 12, $21 \mathrm{~mm}$ in 12, and $23 \mathrm{~mm}$ in six patients (not applicable in one patient). 


\section{Statistical analysis}

Values are presented as means \pm standard deviation. Data were analysed using SPSS version 17.0 software (SPSS Inc., Chicago, IL, USA). Group differences were determined using t-tests for continuous variables and chi-squared test or Fisher's exact tests for categorical variables. Logistic regression was performed for the multivariate analysis for the risk factors for residual MR. Receiver operating characteristic (ROC) curve analysis was used to determine the cut-off value of the risk factor. Kaplan Meier analysis with log-rank test was used for freedom from all-cause death and cardiac related death. $\mathrm{P}$ values under 0.05 were considered statistically significant.

\section{Results}

\section{Change of MR grade}

The MR grade remained moderate in all patients in the residual MR group ( $n=11,33 \%)$. It was improved to none in three and mild in 19 patients in the improved MR group $(n=22,67 \%)$. There were no patients with postoperative severe MR before discharge.

\section{Preoperative echocardiographic parameters}

Table 1 shows the preoperative characteristics of the patients with residual moderate MR and those with improved post-surgery MR. Although most of the patients had some calcifications in the mitral annulus (73\%) and mitral leaflet thickening (52\%), these did not correlate with the residual MR. The incidence of chronic atrial fibrillation tended to be higher in the residual MR group (45\%) than in the improved MR group (14\%; $\mathrm{P}=0.08)$. The left atrial diameter was significantly larger in the residual MR group (51 $\pm 7 \mathrm{~mm}$ ) than in the improved MR group (46 $\pm 5 \mathrm{~mm}$; $\mathrm{P}=0.049$ ). The mean pressure gradient at the AV was significantly smaller in the residual MR group (52 $\pm 18 \mathrm{mmHg}$ ) 
than in the improved MR group (69 $\pm 22 \mathrm{mmHg}$ P $=0.043)$. LV size or fractional shortening did not differ between the groups.

The use of bio-prosthetic valves and valve sizes were not different between the groups. The postoperative peak AV velocity was comparable between residual MR and improve MR group (3.0 \pm 0.6 vs. $2.9 \pm 0.6 \mathrm{~m} / \mathrm{s}$, respectively; $\mathrm{P}=0.65$ ).

\section{Predictors for residual MR}

In multivariate analysis using the two parameters (left atrial diameter and mean pressure gradient), mean pressure gradient was the independent predictor for residual MR ( $\mathrm{P}=0.049)$. In the ROC curve analysis of the gradient, the area under the ROC curve was $0.713(\mathrm{P}=0.06)$. Furthermore, the ratio of left atrial diameter and mean AV pressure gradient was significantly greater in the residual MR group than in the improved MR group (1.09 \pm 0.41 vs. $0.73 \pm 0.21$, respectively; $\mathrm{P}=0.022)$, Fig. 1 .

Fig. 2 shows the ROC curve analysis of the ratio of left atrial diameter (mm) and mean AV pressure gradient (mmHg) for the prediction of residual MR. A ratio of left atrial diameter (mm) and mean AV pressure gradient (mmHg) greater than 0.9 predicted the residual MR with a sensitivity of $70 \%$ and specificity of $74 \%$ (area under the ROC curve, $0.779 ; \mathrm{P}=0.015$ ), which was better than in the mean pressure gradient alone.

Fig. 3 indicates the scatter plots of left atrial diameter and mean AV pressure gradient. There was no correlation between the two parameters. In most of the patients with high ratio (>1.0), moderate MR did not improve after surgery. When a patient has a left atrial diameter of $45 \mathrm{~mm}$ and a mean $\mathrm{AV}$ pressure gradient of $45 \mathrm{mmHg}$, the ratio calculated is 1.0. In this case, preoperative moderate MR may not improve after SAVR.

Mitral valve thickening was observed in 9 (53\%) and 6 (60\%) patients with lower $(<0.9)$ and higher ratio ( $>0.9)$, respectively $(\mathrm{P}=1.00)$. Small mitral leaflet calcification was observed in 
only one case in the lower ratio group. The thickening and small calcification of the leaflets correlated neither to the ratio nor to residual MR.

\section{Late outcomes}

There was no hospital mortality in both groups. One person in each group was readmitted to the hospital due to heart failure: one person in the residual MR group, two years after SAVR, and one in the improved MR group, eight years after SAVR. Both were not related to worsened MR. Late follow-up echocardiography (>1 year post surgery; $60 \pm 51$ months) was available in 17 patients; MR grades did not significantly change $(\mathrm{P}=0.33)$. One patient in the residual MR group presented with severe MR after fluid retention due to renal failure four years after SAVR. In the improved MR group, one patient on long-term hemodialysis presented with severe deterioration of the bioprosthetic valve (AV area: $0.53 \mathrm{~cm}^{2}$ ) and severe MR, two years after SAVR.

Fig. 4 shows the freedom from all-cause death (Fig. 4A) and cardiac related death (Fig. 4B). The median follow-up period was 28 months. Five-year survival rate was significantly worse in the residual MR group than in the improved MR group (53 and 100\%, respectively; Log Rank P $=0.037)$. However, the causes of deaths were not cardiac-related (gastric carcinoma, colon perforation, and mediastinitis). The survival rate was significantly lower in hemodialysis patients than in non-hemodialysis patients (3-year survival was $80 \%$ and $92 \%$, respectively; $\mathrm{p}=0.032$ ).

\section{Discussion}

We demonstrated that in patients with severe AS and functional moderate MR before surgery, MR did not decrease in 33\% of patients after the surgery. The left atrial diameter was significantly larger and the mean AV pressure gradient smaller in the residual MR group than in the improved 
MR group. A ratio of left atrial diameter (mm) and mean AV pressure gradient (mmHg) greater than $0.9(\mathrm{~mm} / \mathrm{mmHg})$ predicted residual MR well.

\section{Pathophysiology of MR in severe AS}

In a review by Lancellotti $\mathrm{P}$ et al. two factors in the pathophysiology of MR in severe AS were reported: 1) increase in left ventricle (LV) to left atrial pressure gradient and 2) mitral valve deformation after LV remodelling by pressure overload (2). Furthermore, MR decreases forward stroke volume, thus reducing the AV pressure gradient, which makes the detection of AS more difficult (low-flow, low-gradient AS). Atrial fibrillation, which is common in patients with AS and MR, may also reduce forward stroke volume. Lee PH et al. reported that in AS patients with a low mean pressure gradient ( $<40 \mathrm{mmHg}$ ), those with MR tend to have more clinical events than those without MR (6). They attributed the high incidence of events to the presence of MR itself or to underestimated AS severity. In the current study, 90\% of the patients had high mean pressure gradients ( $>40 \mathrm{mmHg}$ ). In only one of four patients with a low gradient ( $<40 \mathrm{mmHg}$ ), preoperative MR improved. Further studies are necessary to examine the change of MR status in patients with low-gradient, severe AS.

\section{Predictors for residual MR after AVR}

Ruel et al. examined clinical predictors of prognosis in 107 patients with moderate functional MR before SAVR (7). Left atrial size $>50 \mathrm{~mm}$, preoperative mean AV gradient $<40 \mathrm{mmHg}$, and chronic atrial fibrillation were significant risk factors for postoperative residual moderate MR. Functional MR improved in $70 \%$ of patients without risk factors. These data are in accordance with our current results. In contrast, moderate MR in itself had no independent adverse effect on overall mortality after adjustment for baseline differences. In an analysis of 11,104 patients after transcatheter AV replacement (TAVR) in the US Transcatheter Valve Therapy Registry from 2012 to 2013, 66\% of 
patients with moderate MR at baseline improved before discharge. Patients whose preoperative moderate MR improved had lower heart failure re-hospitalization compared with patients whose MR did not improve (8). In a multivariate analysis, factors associated with persistent MR after TAVR included lower body surface area, increased left ventricular dimension, decreased mean AV gradient, and atrial fibrillation/flutter. Taken together, although moderate MR improves after AVR in more than half of AS patients, it may cause heart failure symptoms later when it does not improve after surgery. A preoperative, low mean AV pressure gradient and atrial fibrillation would be risk factors for residual MR after AVR.

In the current study, we have proposed a combination of two parameters (left atrial size and AV pressure gradient) as a predicting parameter for the change of functional MR in AS. Although we did not assess the mitral annular area, large left atrial size may indicate dilated mitral annulus (Carpentier type I). Lower AV pressure gradient may correspond to the factor 1) in the Lancellotti's review: less LV to left atrial pressure gradient (2). Therefore, in terms of the change in pressure gradient, the effect of AVR on moderate MR would be less in those with lower MPG than in those with higher mPG. Prosthesis-patient mismatch may also influence residual MR. Although the effective orifice area of artificial valve was not applicable in most cases, the postoperative peak AV velocity was comparable between residual MR and improved MR group. Therefore, the postoperative pressure gradient may not have influenced residual MR. We could not attribute residual MR to the factor 2) i.e. LV remodeling because the LV dimensions and fractional shortening were within normal range and not different between the groups in this cohort.

\section{Limitations}

Several limitations of the present study should be addressed. First, the number of subjects was small. The incidence of moderate MR was only $15 \%$ in this SAVR cohort and $26 \%(17 / 65)$ in candidates for TAVR from 2016 to 2018 in our institution (data not shown). Further study including 
more patients would be necessary to use the cut-off value of LAD/mPG for decision making. Second, the evaluation of MR by echocardiography was not based on regurgitation volume or ratio. However, the evaluation of MR was conducted by blinded, specialized sonographers or cardiologists, and the grouping of the patients would therefore be objective. Third, echocardiographic data in the late period were available only in half of the patients. This might have caused underestimation of the recurrence of MR. Nonetheless, this study focused mainly on the early effects of valve replacement on MR. Further studies are necessary to evaluate late MR and artificial valve functions.

\section{Conclusions}

In patients with severe AS and moderate MR, a high ratio of preoperative left atrial diameter and mean AV pressure gradient would be a parameter predicting residual moderate MR post-SAVR. 


\section{Acknowledgements}

We would like to thank Editage (www.editage.jp) for English language editing.

\section{Disclosures}

The authors declare no conflicts of interest, financial or otherwise. 


\section{References}

1. Steiner JM, Cooper S, Kirkpatrick JN. Palliative care in end-stage valvular heart disease. Heart. 2017; 103: 1233-7.

2. $\quad$ Unger P, Dedobbeleer C, Van Camp G, Plein D, Cosyns B, Lancellotti P. Mitral regurgitation in patients with aortic stenosis undergoing valve replacement. Heart. 2010; 96: 9-14. 3. Barreiro CJ, Patel ND, Fitton TP, Williams JA, Bonde PN, Chan V, et al. Aortic valve replacement and concomitant mitral valve regurgitation in the elderly: impact on survival and functional outcome. Circulation. 2005; 112: I443-7.

4. Nishimura RA, Otto CM, Bonow RO, Carabello BA, Erwin JP, 3rd, Guyton RA, et al. 2014 AHA/ACC guideline for the management of patients with valvular heart disease: executive summary: a report of the American College of Cardiology/American Heart Association Task Force on Practice Guidelines. Journal of the American College of Cardiology. 2014; 63: 2438-88.

5. Zoghbi WA, Enriquez-Sarano M, Foster E, Grayburn PA, Kraft CD, Levine RA, et al. Recommendations for evaluation of the severity of native valvular regurgitation with twodimensional and Doppler echocardiography. J Am Soc Echocardiogr. 2003; 16: 777-802. 6. Lee PH, Hong JA, Sun BJ, Han S, Park S, Jang JY, et al. Impact of Significant Mitral Regurgitation on Assessing the Severity of Aortic Stenosis. Journal of the American Society of Echocardiography : official publication of the American Society of Echocardiography. 2018; 31: 26-33.

7. Ruel M, Kapila V, Price J, Kulik A, Burwash IG, Mesana TG. Natural history and predictors of outcome in patients with concomitant functional mitral regurgitation at the time of aortic valve replacement. Circulation. 2006; 114: I541-6. 
8. Mavromatis K, Thourani VH, Stebbins A, Vemulapalli S, Devireddy C, Guyton RA, et al. Transcatheter Aortic Valve Replacement in Patients With Aortic Stenosis and Mitral Regurgitation. The Annals of thoracic surgery. 2017; 104: 1977-85. 


\section{Figure legends}

Figure 1. Ratio of left atrial diameter and mean aortic valve pressure gradient in residual and improved mitral regurgitation groups

Figure 2. Receiver operating characteristic curve analysis for the prediction of residual mitral regurgitation after isolated aortic valve replacement in patients with severe aortic valve stenosis. AUC, area under the curve; LAD, left atrial diameter (mm); mPG, mean aortic valve gradient (mmHg)

Figure 3. Scatter plots of left atrial diameter and mean aortic valve pressure gradient. The solid line indicates that the ratio of $\mathrm{LAD} / \mathrm{mPG}$ is 0.9 . The dotted line indicates that the ratio of $\mathrm{LAD} / \mathrm{mPG}$ is 1.0. LAD, left atrial diameter (mm); mPG, mean aortic valve gradient (mmHg).

Figure 4. Freedom from all-cause death (A) and cardiac related death (B). MR, mitral regurgitation. 
Table 1. Preoperative echocardiographic parameters of patients with residual moderate MR and those with improved post-surgery MR

\begin{tabular}{|c|c|c|c|c|}
\hline & $\begin{array}{l}\text { Total } \\
(\mathrm{n}=32)\end{array}$ & $\begin{array}{l}\text { Residual MR } \\
\qquad(\mathrm{n}=11)\end{array}$ & $\begin{array}{l}\text { Improved MR } \\
\qquad(\mathrm{n}=21)\end{array}$ & $\begin{array}{c}\mathrm{P} \\
\text { value }\end{array}$ \\
\hline Age & $74 \pm 7$ & $73 \pm 7$ & $74 \pm 7$ & 0.58 \\
\hline Male & $10(30 \%)$ & 5 & 5 & 0.25 \\
\hline Chronic atrial fibrillation & $8(24 \%)$ & 5 & 3 & 0.09 \\
\hline \multicolumn{5}{|l|}{ Transthoracic echo parameters } \\
\hline Aortic valve area, $\mathrm{cm}^{2}$ & $0.72 \pm 0.28$ & $0.83 \pm 0.30$ & $0.68 \pm 0.25$ & 0.16 \\
\hline Mean aortic valve pressure gradient, $\mathrm{mmHg}$ & $63 \pm 22$ & $52 \pm 18$ & $69 \pm 22$ & 0.047 \\
\hline Aortic valve regurgitation (none/mild/moderate/severe) & $4 / 11 / 14 / 3$ & $1 / 3 / 6 / 1$ & $3 / 8 / 8 / 2$ & 0.84 \\
\hline Tricuspid valve regurgitation (none/mild/moderate/severe) & $6 / 13 / 12 / 1$ & $1 / 4 / 6 / 0$ & $5 / 9 / 6 / 1$ & 0.44 \\
\hline Left ventricular end-diastolic dimension, mm & $49 \pm 6$ & $50 \pm 7$ & $48 \pm 6$ & 0.51 \\
\hline Left ventricular end-systolic dimension, mm & $32 \pm 7$ & $32 \pm 7$ & $32 \pm 8$ & 0.99 \\
\hline Left atrial diameter, mm & $47 \pm 6$ & $51 \pm 7$ & $46 \pm 5$ & 0.049 \\
\hline Interventricular septal thickness, mm & $14 \pm 3$ & $15 \pm 5$ & $14 \pm 2$ & 0.25 \\
\hline Posterior wall thickness, mm & $12 \pm 2$ & $11 \pm 1$ & $12 \pm 2$ & 0.32 \\
\hline Fractional shortening, \% & $35 \pm 10$ & $35 \pm 6$ & $34 \pm 11$ & 0.91 \\
\hline Systolic pulmonary pressure, mmHg & $40 \pm 16$ & $35 \pm 16$ & $42 \pm 16$ & 0.31 \\
\hline Mitral valve thickening & $16(53 \%)$ & 8 & 8 & 0.14 \\
\hline Mitral annular calcification & 23 (70\%) & 7 & 16 & 0.68 \\
\hline
\end{tabular}

Values \pm standard deviation; MR, mitral regurgitation 
Fig. 1

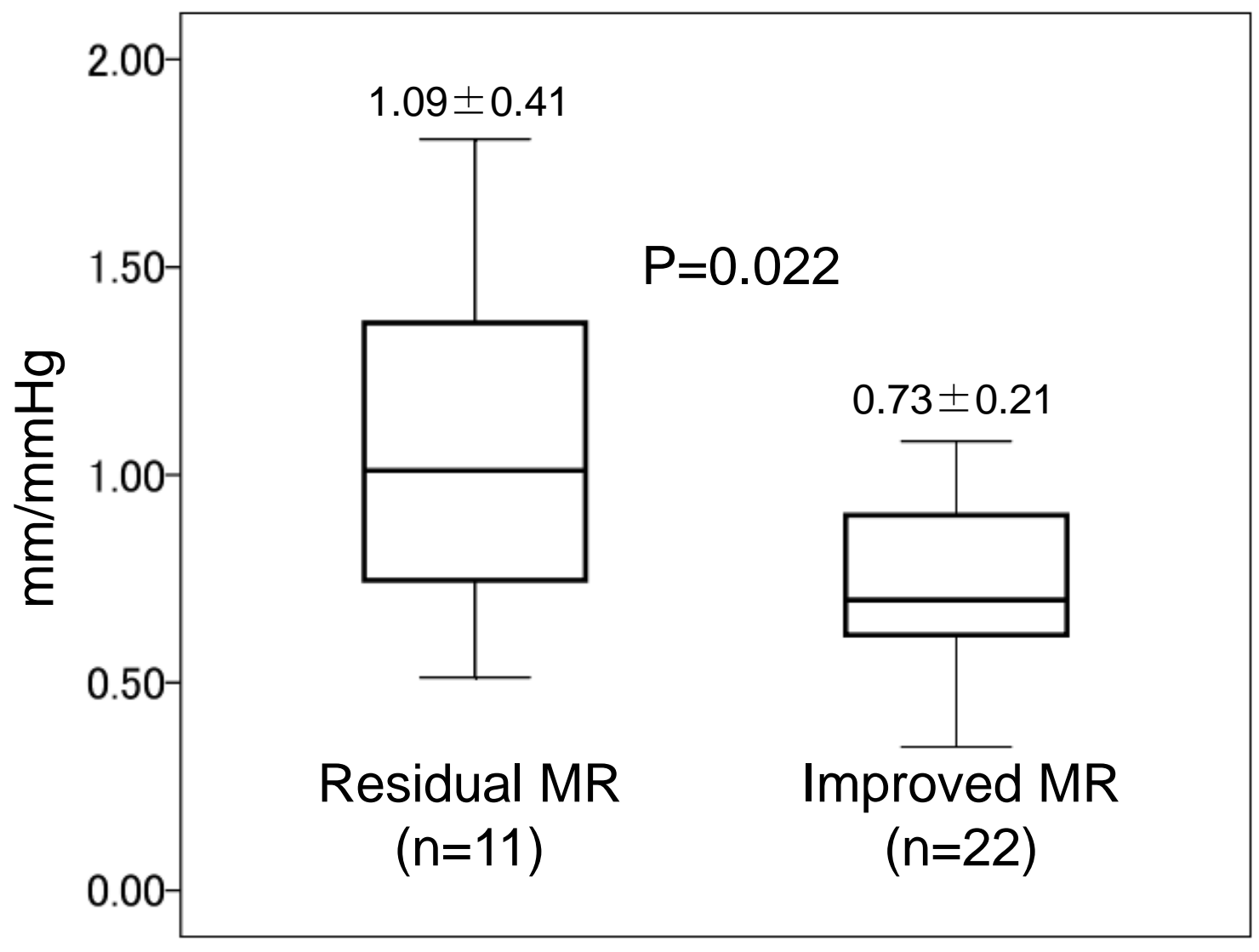


Fig. 2

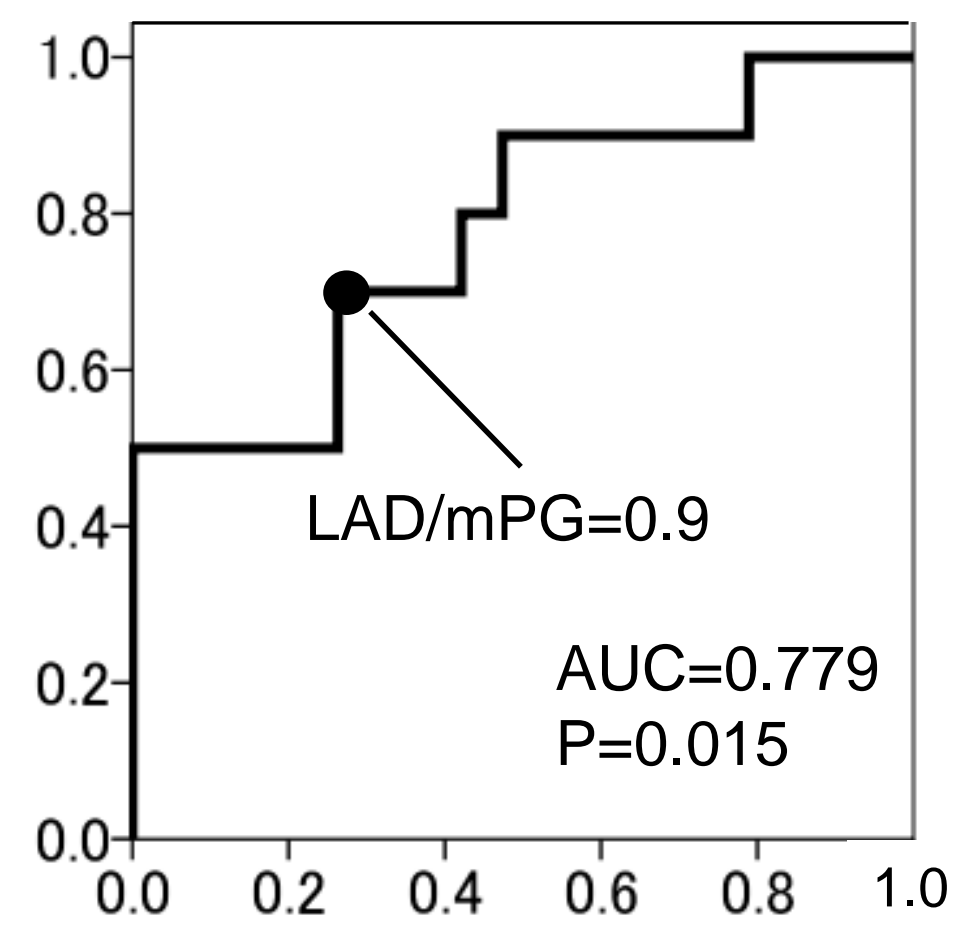


Fig. 3

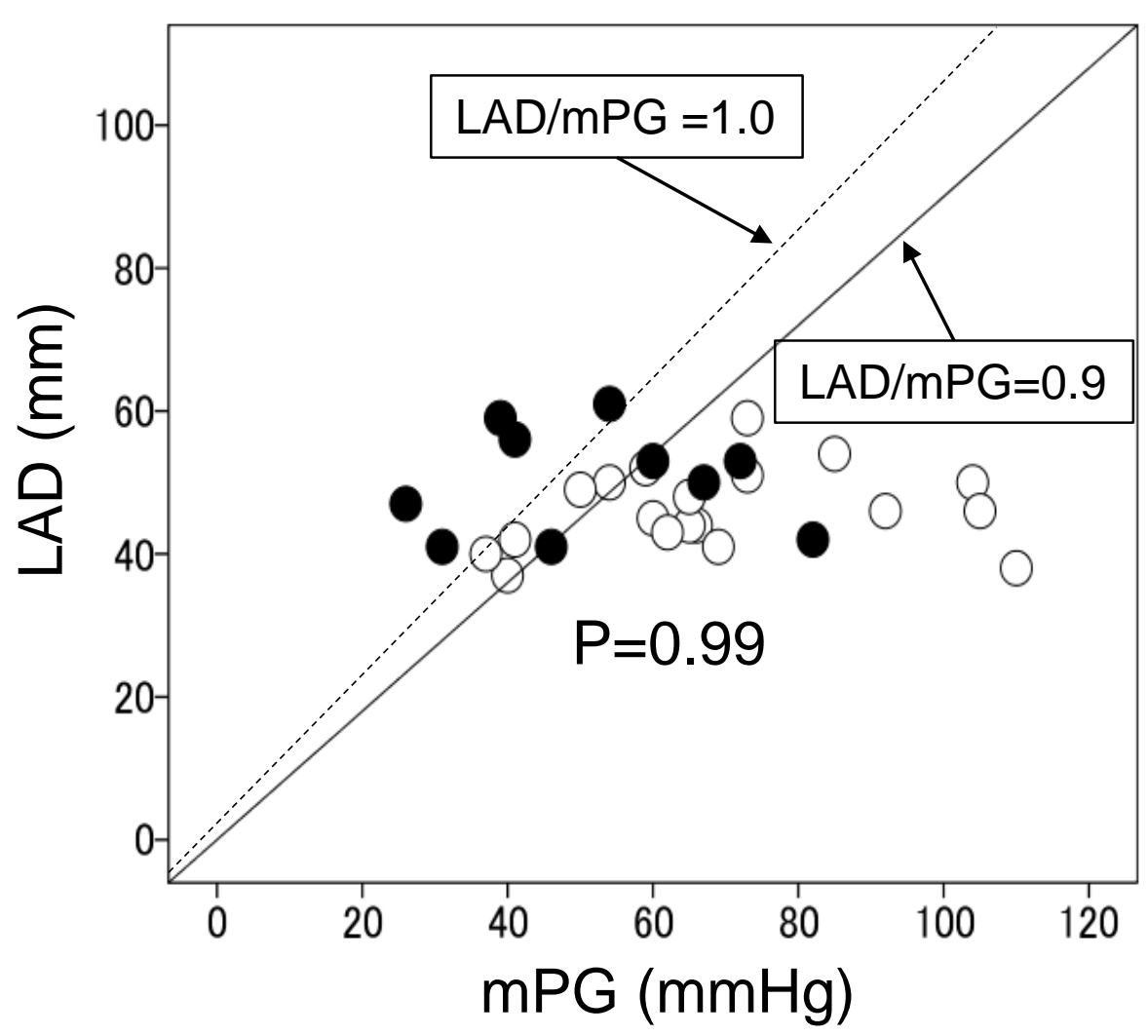


Fig. 4

A

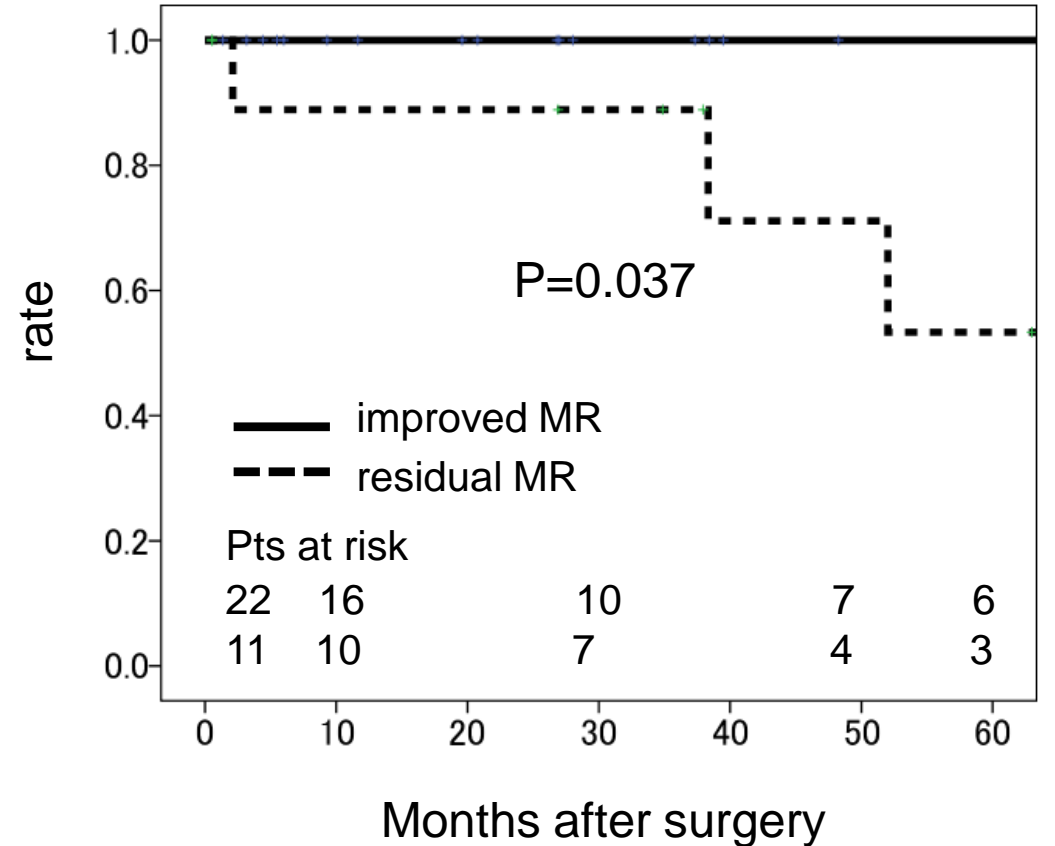

B

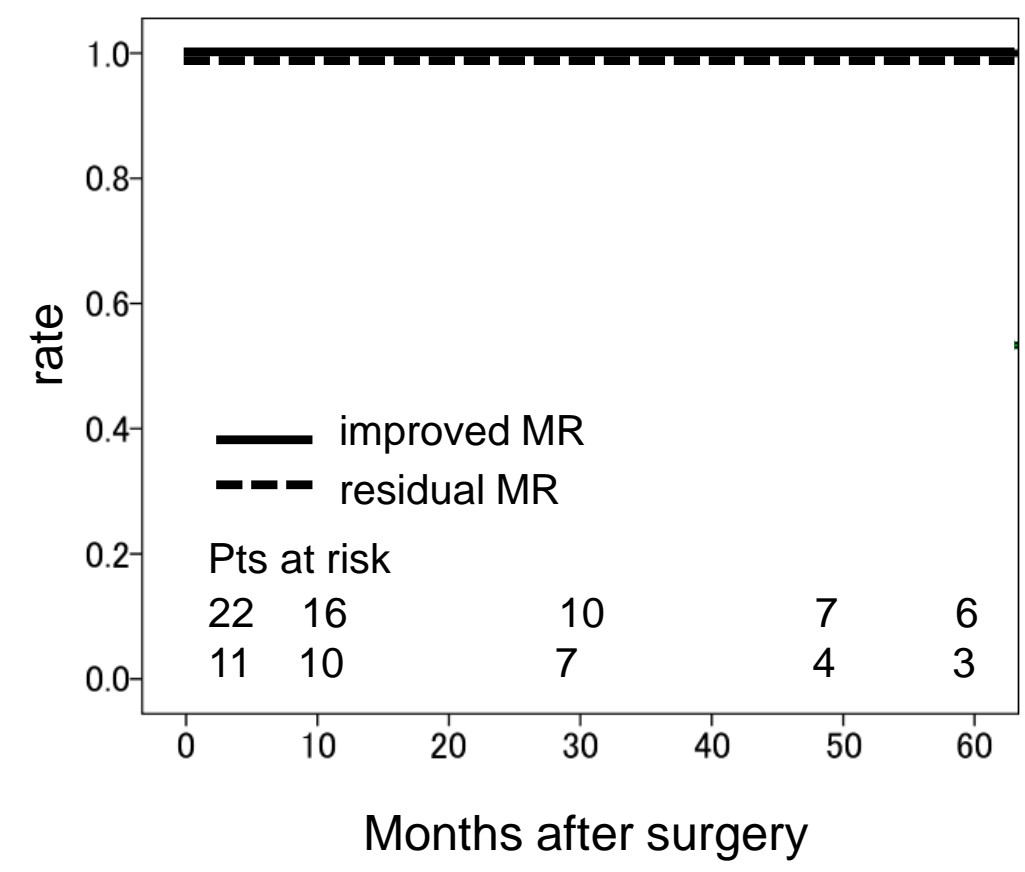

